\title{
PREFERENCIAS Y ACTITUD DISCENTE HACIA LOS JUEGOS DE MESA: ANÁLISIS MULTIVARIANTE
}

\author{
Student Preferences and Attitude toward Board Games: Multivariate Analysis
}

\author{
Sebastián Cristóbal Araya-Pizarro ${ }^{1}$ \\ ORCID: 0000-0002-5857-8441 \\ ${ }^{1}$ Universidad de La Serena, Chile \\ Correo: saraya@userena.cl
}

Recibido: 14/01/2021

Aceptado: 05/04/2021

Resumen: El propósito de este estudio es analizar las preferencias y la actitud discente hacia los juegos de mesa entre estudiantes universitarios. La investigación contó con la participación de estudiantes de la asignatura Gestión de Recursos Humanos, del grado de Administración de Empresas de la Universidad de La Serena (Chile), así como expertos en juegos de mesa, educación y gestión de recursos humanos. Se realizó un análisis estadístico descriptivo y multivariante (modelo composicional multiatributo), para el que se establecieron dos etapas secuenciales: una fase exploratoria-descriptiva para identificar los atributos más importantes de los juegos de mesa para la enseñanza de la asignatura, seguida de una fase explicativa y corte transversal para medir las preferencias y percepción discente hacia los juegos de mesa. Los resultados mostraron la predisposición favorable hacia los juegos de mesa, a los que consideran idóneos para desarrollar habilidades sociales, habilidades para la resolución de problemas y habilidades analíticas. Además, se determinó que los juegos preferidos son aquellos con alta interacción entre participantes, de tipo colaborativo, con un tiempo de dedicación máximo de 90 minutos y con un nivel de complejidad medio. El contraste de preferencias según sexo reveló que los hombres prefieren los juegos competitivos, de alta interacción y complejidad, mientras que las mujeres prefieren los juegos colaborativos, de interacción y complejidad media. Se concluye que los juegos de mesa representan una metodología con alto potencial de aplicabilidad en la enseñanza y aprendizaje de la gestión de recursos humanos, pero que debe adaptarse a las preferencias del estudiantado.

Palabras clave: juego educativo; estrategias educativas; educación superior; gestión; recursos humanos; análisis multivariado.

\begin{abstract}
The purpose of this study is to analyze the preferences and attitudes towards board games among university students. The research counted with the participation of students of the Human Resources Management course, of the Business Administration degree at the Universidad de La Serena (Chile), as well as experts in the fields of board games, education, and human resources management. A descriptive and multivariate statistical analysis (multiattribute compositional model) was performed, for which two sequential stages were established: an exploratorydescriptive phase to identify the most important attributes of board games for the teaching of the discipline, followed by an explanatory and cross-sectional phase to measure student preferences and perception towards board games. The results showed a favorable predisposition towards board games, which were considered suitable for developing social, problem-solving, and analytical skills. In addition, it was determined that the preferred games are those with high interaction among participants, of a collaborative type, with a maximum dedication of 90 minutes and a medium level of complexity. The contrast of preferences according to sex revealed that men prefer competitive games, with high interaction and complexity, while women prefer collaborative games, with medium interaction and complexity. It is concluded that board games represent a methodology with a high potential for applicability in the teaching and learning of human resources management, but that it should be adapted to the preferences of the students.
\end{abstract}

Keywords: educational games; educational strategies; higher education; management; human resources; multivariate analysis. 


\section{Introducción}

Hoy el mundo contemporáneo precisa de profesionales integrales, cuya formación incorpore no solo el desarrollo de conocimientos teóricos, sino también de actitudes y habilidades que les permitan desempeñarse en entornos altamente dinámicos y competitivos (Araya-Pizarro, 2019). Por lo tanto, las instituciones de educación, sobre todo las universidades, requieren incorporar modelos de enseñanza y herramientas pedagógicas innovadoras, ajustadas a esta realidad, y que posibiliten desarrollar el potencial del estudiantado en un sentido multidimensional (cognitivo, emocional y valórico) y bajo un contexto estimulante. Esto trae consigo la responsabilidad de las organizaciones educativas de incluir estrategias que aumenten la motivación del alumnado y el compromiso de facilitar todos los recursos y herramientas que favorezcan el aprendizaje autónomo y significativo (González et al., 2017; Ortiz-Colón et al., 2018).

Es por esto que las metodologías activas y las estrategias pedagógicas lúdicas, como los juegos, adquieren gran connotación, pues se les atribuyen numerosas ventajas sobre los métodos tradicionales de enseñanza y aprendizaje. Por una parte, desinhiben, relajan, motivan y posibilitan una comunicación real al interior del aula. Y, por otra, permiten el desarrollo de dimensiones afectivas, que junto con la aplicación de diversos procesos cognitivos fundamentales, como deducir, inferir y formular hipótesis, estimulan una reacción activa, crítica, reflexiva y creativa del estudiantado (Sánchez, 2010; Urquidi y Calabor, 2014). Ello explica el surgimiento del interés por el aprendizaje basado en juegos (Game-Based Learning o GBL) y la gamificación como estrategia didáctica para la educación. Es decir, técnicas que permitan integrar los aspectos de la dinámica del juego en contextos no lúdicos (Ortiz-Colón, et al., 2018).

Los juegos de mesa se definen como un sistema matemático interactivo, hecho concreto, usado para contar una historia (Daviau, 2011). Se presentan como un esquema mental y físico amplio para los jugadores. Una compilación de dinámicas y mecánicas, que no sólo aportan entretenimiento, sino que mantienen la mente activa de los jugadores, incrementando la capacidad de aprender y de desarrollar el pensamiento crítico (Melo y Hernández, 2014; Victoria-Uribe et al., 2017).

En el ámbito educativo, existen diversos artículos que muestran la utilidad de los juegos de mesa para el aprendizaje del estudiante (Hassinger-Das et al., 2017; Rogerson et al., 2018) encontrándose variadas investigaciones aplicadas, tanto en la educación escolar (Díez et al., 2017; Pérez, 2016) como en la educación superior (Corchuelo-Rodriguez, 2018; Esnaola et al., 2014; 
Hernández-Horta et al., 2018; Zepeda-Hernández et al., 2016). En general se plantea que la utilización exitosa de los juegos implica el involucramiento emocional de los participantes y su motivación para alcanzar las metas (Burke, 2016) por lo que su efectividad estaría supeditada al desarrollo de una planificación exhaustiva y profunda acorde a las preferencias del alumnado.

Dado lo anterior, nace la idea de revisar la aplicación de los juegos de mesa como metodología de enseñanza en educación superior, de acuerdo con la percepción del alumnado. En particular se busca responder: ¿cuál es la disposición discente hacia los juegos de mesa? y ¿qué atributos explican la predilección por este tipo de juego? De este modo se plantea por objetivo analizar las preferencias y la actitud del estudiantado, de la asignatura Gestión de Recursos Humanos, por los juegos de mesa. Para ello, se utiliza un modelo composicional multiatributo que mide la importancia relativa de diferentes atributos de juego, definidos en base a encuestas a expertos y estudiantes del grado de Administración de Empresas. Cabe mencionar que la investigación se aplica en estudiantes de la asignatura Gestión de Recursos Humanos, cuyo perfil supone el desafío de desarrollar aptitudes de autonomía e iniciativa en la toma de decisiones, así como capacidad para trabajar en equipos y resolver problemas relacionados a habilidades de liderazgo, gestión y espíritu emprendedor. Competencias que podrían ser potenciadas a través del juego (Hamari et al., 2014).

En relación con lo antes expuesto, la investigación se justifica por la necesidad de descubrir estrategias y metodologías de enseñanza que contribuyan a aumentar la motivación y el aprendizaje efectivo del estudiantado; factores condicionantes del desempeño académico (Cerda et al., 2017) y desafíos de larga data para la educación (Dichev y Dicheva, 2017). Especialmente de aquellas metodologías que favorecen un rol activo del discente, el aprendizaje significativo, la colaboración y la autonomía (Silva y Maturana, 2017). Como indican Taspinar et al. (2016) enseñar a los estudiantes de una manera agradable y exitosa requiere, cada vez más, la inclusión de elementos interactivos en la mezcla de componentes didácticos. En este contexto, el estudio genera conocimiento sobre los componentes de juego preferidos por el alumnado universitario (importancia relativa) y, por tanto, devela aquellos aspectos de jugabilidad que resultarían más plausibles para lograr su interés y motivación. Estos atributos representarían la base (criterios) para la selección de los juegos que pretendan implementarse en la asignatura de Gestión de Recursos Humanos y materias afines. A lo que se suma, el aporte que representa la generación de resultados en un contexto diferente como lo es el latinoamericano, y particularmente, de Chile. 
Por otro lado, cabe destacar la contribución de la metodología de análisis aplicada en el estudio: modelo multiatributo de elección discreta. Esta técnica, a diferencia de los métodos tradicionales, no consulta directamente por la importancia que se otorga a cada atributo individual de juego; sino que mediante un proceso de trade-off (clasificación de cada opción de juegos de mesa) la persona devela de manera tácita la relevancia que asigna a los atributos que la componen (interacción, complejidad, duración y formato) y los niveles de utilidad que les reporta, lo que conlleva a examinar el proceso decisional de manera más realista (Hair et al., 2014).

El estudio hipotetiza que las preferencias por un juego de mesa dependen de cuatro atributos de juego destacados por la literatura y confirmados por los resultados obtenidos del trabajo de campo de la investigación: tiempo de dedicación (Gómez, 2020; Zeng et al., 2017), complejidad (Urquidi y Calabor, 2014; Zeng et al., 2017), formato de juego (Victoria-Uribe et al., 2017; Zagal et al., 2006) y nivel de interacción con otros jugadores (Ortiz-Colón et al., 2018; Swartout y van Lent, 2003; Zepeda-Hernández et al., 2016). Específicamente, la predilección por un juego de mesa se incrementaría cuando el juego es participativo, no complejo, de corta duración y colaborativo. Por lo demás, cabría encontrar diferencias entre el perfil de juego preferido y las características del alumnado (Baek y Touati, 2020; Curlango et al., 2014; Kimura, 2004).

Se espera que los resultados de la investigación permitan establecer las pautas para la selección de los juegos apropiados para la materia, es decir, de aquellos que faciliten lograr los objetivos de aprendizaje planteados en base en las preferencias declaradas por el alumnado (Dichev y Dicheva, 2017).

\section{Metodología}

\section{Diseño del estudio}

La investigación tuvo un enfoque cuantitativo, diseño no experimental, conformada de dos etapas de tipo secuencial: una fase exploratoria-descriptiva, seguida de una fase explicativa y corte transversal. La primera etapa tuvo por propósito identificar los atributos de los juegos de mesa más importantes para la enseñanza de la asignatura Gestión de Recursos Humanos. La segunda etapa, planteó por objetivo medir las preferencias y percepción discente hacia los juegos de mesa. 


\section{Participantes}

La primera fase del estudio contó con la participación de tres especialistas en juegos, educación y administración: un doctor en educación y director de un laboratorio de juegos (Argentina); un experto en juegos y propietario de una empresa comercializadora de juegos de mesa (Chile); y un académico, candidato a doctor en educación, magíster en recursos humanos y especialista en juegos de mesa (Chile). Además, para la confirmación y establecimiento de los atributos y niveles de juego, participaron 25 estudiantes del grado de administración de empresas de la Universidad de La Serena (ULS).

Para la segunda fase, se contó con 42 estudiantes del mismo grado que cursan la asignatura Gestión Estratégica de Recursos Humanos. Esta posee seis competencias declaradas en su programa: 1) Integrar conocimientos disciplinarios de recursos humanos para la administración y gestión de empresas o el desarrollo de proyectos y emprendimientos; 2) Capacidad para administrar y gestionar empresas, negocios y funciones organizacionales tanto en el sector público como privado; 3) Demostrar una actitud emprendedora, un enfoque prospectivo y una visión holística, en la generación de nuevos negocios tanto dentro como fuera de la organización; 4) Capacidad de trabajar y liderar equipos en pos del logro de los objetivos grupales y con énfasis en el mejoramiento continuo, adecuándose a entornos cambiantes y aprovechando el talento de las personas; 5) Justifica la importancia de la ética, y es capaz enfocar su trabajo hacia la responsabilidad social y 6) Explicar el liderazgo como una capacidad de influir en las personas e impactar en los resultados de las organizaciones. Además, busca desarrollar diversas competencias transversales: compromiso ético, capacidad para organizar y planificar el tiempo, conocimientos sobre el área de estudio y la profesión, capacidad de comunicación oral y escrita, habilidades para buscar, procesar y analizar la información, capacidad crítica y autocrítica, capacidad para tomar decisiones, capacidad para trabajo en equipo, habilidades interpersonales, capacidad para motivar y conducir hacia objetivos comunes y compromiso con el medio sociocultural.

La asignatura incluye como principales estrategias metodológicas la clase expositiva, el estudio de casos y el aprendizaje cooperativo. Por otro lado, los métodos de evaluación son la presentación de portafolios, ensayos, disertaciones y elaboraciones grupales de propuestas de intervención. La asignatura se imparte en el séptimo nivel del grado de administración, tiene una duración de un semestre, con una carga estándar de 4 créditos, distribuidos en 3 horas directas de cátedra semanal que se organizan en dos sesiones de 90 minutos cada una. 


\section{Técnicas de recolección de datos}

Las técnicas para la recolección de datos fueron tres encuestas ad hoc aplicadas a expertos y estudiantes. Todas realizadas durante los meses de septiembre y octubre de 2018 (Figura 1).

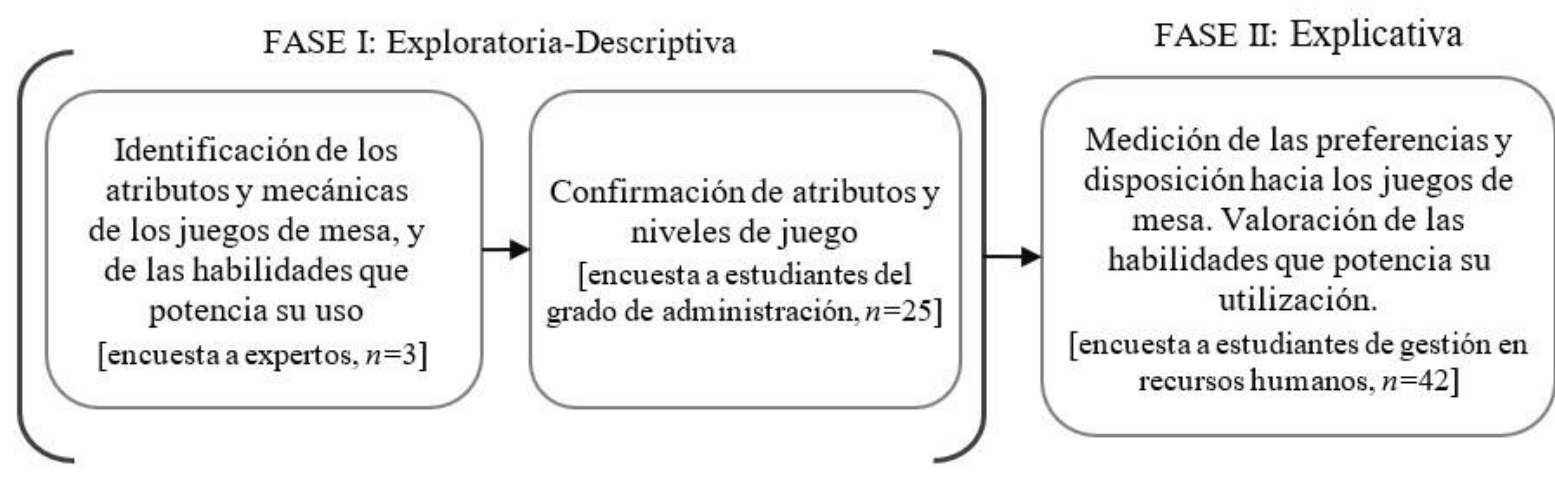

Figura 1. Fases, participantes y técnicas del estudio. Elaboración propia (2021)

La encuesta a los expertos buscó determinar las características (atributos) y mecánicas de juego que resultan significativas para el aprendizaje. El instrumento contó con un listado de atributos de juego que debieron calificar bajo una escala Likert de cinco puntos, según su nivel de importancia ( $1=$ muy bajo hasta $5=$ muy alto) y su contribución a la asignatura. Conjuntamente, los especialistas fueron consultados respecto de las habilidades y capacidades que consideran son potenciadas por los juegos de mesa. Sus respuestas, ajustadas y clasificadas en base a la propuesta de capacidades y habilidades del modelo Tuning América Latina (Beneitone et al., 2007), fueron posteriormente sometidas a evaluación por parte del alumnado (fase II).

La encuesta para estudiantes del grado de administración se aplicó con el propósito de confirmar la selección de los atributos y niveles para los juegos de mesa. Este instrumento evaluó, a través de una escala Likert de 5 puntos (1=muy bajo y $5=$ muy alto) la importancia de variados atributos de los juegos de mesa para el proceso de enseñanza-aprendizaje: tipo, formato, duración de una partida, complejidad, ambiente, interacción, participantes, historia, entre otros.

Finalmente, el cuestionario de la segunda fase de investigación contó de tres secciones. La primera parte incorporó siete preguntas de caracterización del estudiantado, en cuanto al sexo (hombre, mujer), tipo de educación escolar (privada, subvencionada, pública), años de edad (menos de 21, entre 21 y 23, entre 24 y 26 y más de 26), ciudad de origen (La Serena, otra), frecuencia de juego (diario, semanal, mensual, anual, nunca), disposición a participar de juegos de mesa (baja, media y alta) y aporte percibido en cuanto a las habilidades que potencian los juegos de mesa (organizativas, meta-cognitivas, memorización, resolución de problemas, creativas, 
analíticas, para la toma de decisiones y sociales). La segunda sección contó de catorce preguntas bajo una escala de Likert de 5 puntos (1=total desacuerdo a 5=total acuerdo), enfocadas en identificar la valoración sobre la contribución percibida de los juegos de mesa al proceso de enseñanza y aprendizaje, y la disposición discente hacia la aplicación de distintas mecánicas de juego. Finalmente, la tercera parte evaluó las preferencias respecto a cuatro atributos y nueve niveles de juego, presentados a través de nueve alternativas (tarjetas o perfiles) hipotéticas.

\section{Técnicas de análisis de datos}

Las respuestas obtenidas de las dos encuestas aplicadas en la fase exploratoria-descriptiva fueron examinadas a través de la construcción de tablas de frecuencias y el cálculo de estadísticos descriptivos de tendencia central (modas, promedios y medianas). La triangulación de los datos permitió determinar las variables que configuraron los perfiles para la medición de las preferencias por juegos de mesa para la enseñanza de la gestión de recursos humanos.

Por otra parte, para analizar los atributos de juego preferidos por los estudiantes, se utilizó el modelo de valorización multiatributo conocido como Análisis Conjunto. Esta técnica tiene una relación directa con la teoría de la demanda de Lancaster (Lancaster, 1966) pues considera que los bienes se componen por diversos atributos que no pueden desagruparse fácilmente de modo que, cuando se selecciona una determinada opción, se está optando por todo el conjunto de características que la constituyen. Es decir, las personas evalúan el valor de un producto o servicio (utilidad total) combinando el valor individual que le reporta cada atributo.

La ecuación matemática, correspondiente al modelo aditivo, utilizada en el estudio fue:

$$
U_{t}=\alpha+\sum_{i=1}^{l} \sum_{j=1}^{k_{i}} \beta_{i j} x_{i j}+\varepsilon_{t}
$$

Donde $U_{t}$ es la variable dependiente, en este caso el orden de preferencia dada por la combinación $\mathrm{t}$ de niveles del atributo de juego, $\alpha$ corresponde a la constante de regresión, $\varepsilon$ representa el error de estimación, $\beta_{\mathrm{ij}}$ es la utilidad parcial generada por el j-ésimo nivel del i-ésimo atributo, $\mathrm{x}_{\mathrm{ij}}$, el cual, si se encuentra presente, adquiere el valor 1, y en caso contrario, adquiere el valor 0. De este modo, en la ecuación, $x_{1 i}$ representa el i-ésimo atributo del j-ésimo nivel de juego.

Las utilidades parciales se calcularon mediante el método de Mínimos Cuadrados Ordinarios (MCO) de acuerdo a los atributos y los niveles de estudio. La importancia de un atributo 
se define en términos del rango de valores parciales $\beta \mathrm{ij}$ en todos los niveles de esa característica y posteriormente se normaliza a fin de ajustar su relevancia en relación a los demás. Así, se puede determinar la importancia relativa del atributo de juego $\left(I R_{i}\right)$ mediante la siguiente fórmula:

$$
I R_{i}=\frac{\text { Rangos de utilidad del atributo de juego }_{i}}{\text { Suma total de rangos de utilidad total de los atributos de juego }} * 100(\%)
$$

Por último, cabe señalar que como medidas de validación del cuestionario (escala Likert) y de bondad de ajuste del modelo se usaron los coeficientes alfa de Cronbach y Tau-b de Kendall, respectivamente. Estos demostraron buenos ajustes, resultando de 0,81 para la escala de apreciación y de 0,78 para la estimación del modelo multivariante.

\section{Resultados}

\section{Identificación de los atributos y niveles de juego}

El análisis de las respuestas de los expertos (porcentaje de importancia) permitió identificar cuatro factores fundamentales de los juegos de mesa: interacción entre los jugadores (93\%), tiempo que requiere una partida (86\%), formato de juego y nivel de complejidad (ambas con 80\%). En general, los especialistas coincidieron en los atributos mencionados, vinculándolos al desarrollo de diferentes habilidades de liderazgo y trabajo en equipo, resolución de problemas y toma de decisiones. Por lo demás, el alumnado confirmó lo antes expuesto, destacando como muy importantes los atributos: tiempo de dedicación (84\%), nivel de complejidad (80\%), formato de juego (80\%) y nivel de interacción con otros jugadores (72\%). De este modo, como resultado del análisis previo, se determinaron los atributos siguientes:

- Interacción entre jugadores: nivel en el que se interactúa con otros jugadores.

- Formato de juego: forma en que se relacionan los jugadores para conseguir una victoria en el juego, pudiendo ser colaborativo (todos los jugadores trabajan en equipo contra el tablero) o competitivo (los jugadores diseñan estrategias que se oponen a las acciones de los demás jugadores).

- Tiempo de una partida: cantidad de tiempo invertido en jugar una partida, que puede ser de una clase o mayor de una clase. 
- Complejidad del juego: nivel de esfuerzo para lidiar con la cantidad de recursos físicos o mentales que se requiere para jugar (concentración, reglamentos, elaboración de estrategias, etc.)

En concreto, los atributos (4) y niveles (10) identificados fueron: Nivel de interacción (bajo, medio y alto), formato de juego (colaborativo, competitivo), tiempo de dedicación ( $<=90$ min, > 90 min.) y nivel de complejidad (bajo, medio y alto). A partir de estas variables se elaboraron nueve opciones de juego, que fueron determinadas mediante un diseño ortogonal, lo que aseguró que todos los atributos y niveles figuraran con idéntica intensidad en los perfiles usados. Y que fueron presentados a los encuestados y encuestadas bajo el enfoque de perfil completo (full profile), es decir, se les expuso las nueve alternativas de juego de manera conjunta, para que manifestaran sus preferencias en base a su jerarquización (ranking del más preferido=1 al menos preferido=9). Las tarjetas usadas en el estudio se muestran en la Tabla 1.

Tabla 1

Tarjetas empleadas para medir las preferencias por juegos de mesa

\begin{tabular}{cllll}
\hline $\begin{array}{c}\text { Alternativa } \\
\text { de juego }\end{array}$ & $\begin{array}{l}\text { Nivel de Interacción } \\
\text { con otros jugadores }\end{array}$ & $\begin{array}{l}\text { Formato de } \\
\text { juego }\end{array}$ & $\begin{array}{l}\text { Tiempo de } \\
\text { dedicación }\end{array}$ & $\begin{array}{l}\text { Nivel de } \\
\text { complejidad }\end{array}$ \\
\hline A & Alto & Colaborativo & 90 min. o menos & Bajo \\
B & Alto & Competitivo & 90 min. o menos & Medio \\
C & Medio & Competitivo & 90 min. o menos & Medio \\
D & Medio & Competitivo & Más de 90 min. & Bajo \\
E & Medio & Colaborativo & 90 min. o menos & Alto \\
F & Bajo & Competitivo & 90 min. o menos & Alto \\
G & Bajo & Competitivo & 90 min. o menos & Bajo \\
H & Alto & Competitivo & Más de 90 min. & Alto \\
I & Bajo & Colaborativo & Más de 90 min. & Medio \\
\hline
\end{tabular}

Fuente. Elaboración propia (2021) 


\section{Caracterización y perfil de juego del alumnado}

Del alumnado encuestado, el $52 \%$ fueron hombres y un $48 \%$ mujeres. La mayoría con edades entre 21 y 23 años (91\%); provenientes de las ciudades de La Serena (38\%), Coquimbo (22\%) y Ovalle (14\%) y procedentes de establecimientos educacionales subvencionados (62\%) y públicos $(36 \%)$.

En relación con los juegos de mesa, se observó que la totalidad del alumnado ha participado de algún juego de este tipo, con frecuencias anual (57\%) y mensual (24\%). Destacaron que las tres principales habilidades que potencian los juegos de mesa son las habilidades para la resolución de problemas (41\%), las habilidades analíticas (38\%) y las habilidades para la toma de decisiones (36\%). Por último, el estudiantado mostró gran disposición (media-alta) por participar de la aplicación de juegos de mesa como estrategia de enseñanza-aprendizaje (90\%).

\section{Actitud discente hacia los juegos de mesa}

Con respecto a la contribución percibida por el alumnado sobre la utilidad de los juegos de mesa hacia el proceso de enseñanza-aprendizaje, se observa una respuesta positiva y homogénea. La mayoría considera que los juegos pueden ser una herramienta de aprendizaje útil (98\%) y que las actividades lúdicas (juegos) facilitan el aprendizaje, haciéndolo entretenido (95\%) ayudando a aprender más $(74 \%)$ y mejor $(88 \%)$.

Ello muestra coincidencia con la inclinación que poseen por clases más didácticas $(86 \%)$ y la predisposición favorable a utilizar los juegos en clases (62\%) para reforzar las habilidades de trabajo en equipo $(60 \%)$. La Tabla 2 resume los resultados obtenidos. 
Tabla 2

Escala de apreciación sobre distintos aspectos de los juegos, valores en porcentaje $(n=42)$

\begin{tabular}{lccc}
\hline Nivel de acuerdo & Bajo & Medio & Alto \\
\hline Considero que los juegos pueden ser una herramienta de aprendizaje útil & 2 & 0 & 98 \\
La actividad lúdica (juego) facilita el aprendizaje haciéndolo entretenido & 2 & 2 & 95 \\
El juego ayuda a que los estudiantes aprendan más & 7 & 19 & 74 \\
Me gustan los juegos que tienen un bajo nivel de complejidad & 45 & 24 & 31 \\
En un juego de equipos, prefiero seguir a un líder, antes que ser uno & 38 & 43 & 19 \\
Prefiero las clases más didácticas & 7 & 7 & 86 \\
Considero que los juegos sencillos y claros facilitan mi proceso de aprendizaje & 26 & 19 & 55 \\
Quisiera que la clase se transforme en un juego inteligente que ayude a aprender & 5 & 14 & 81 \\
Creo que los juegos interactivos facilitan el proceso de aprendizaje & 10 & 10 & 81 \\
Considero que los juegos de diseño llamativo y colorido favorecen el aprendizaje & 2 & 29 & 69 \\
Las actividades lúdicas (juegos) ayudan a los estudiantes a aprender mejor & 2 & 10 & 88 \\
Para mí es importante que las actividades de clases consideren el juego & 7 & 31 & 62 \\
Creo que los juegos pueden reforzar mis habilidades de trabajo en equipo & 5 & 36 & 60 \\
\hline
\end{tabular}

Fuente. Elaboración propia (2021)

En cuanto a las preferencias de los estudiantes hacia la aplicación de distintas mecánicas de juego, se observaron respuestas heterogéneas. A nivel global, se prefieren juegos de complejidad medio-alto (55\%), interactivos (81\%), de estilo llamativo (69\%) y que acentúen el rol activo (liderazgo) del jugador (38\%).

No obstante, es interesante notar las diferencias halladas entre alumnos y alumnas, en cuanto a factores como la complejidad del juego, la atracción hacia los componentes del juego, el nivel de interacción con otros jugadores y las reglas de juego (véase la Figura 2). En este sentido, las mujeres prefieren juegos de mesa con un menor grado de complejidad (60\%), con reglamentaciones sencillas $(80 \%)$ y creen que las características llamativas de los componentes del juego facilitan el desarrollo de metodologías en clase (95\%). Por su parte, los hombres tienen tendencia a preferir juegos de mayor nivel de complejidad (73\%), con reglamentaciones más difíciles $(73 \%)$ y muestran mayor indiferencia frente al uso de componentes atractivos en un juego de fines pedagógicos (55\%). Por lo demás, aunque en general el estudiantado prefiere juegos interactivos, esta característica es más apreciada por las mujeres (95\%) que los hombres (68\%). 


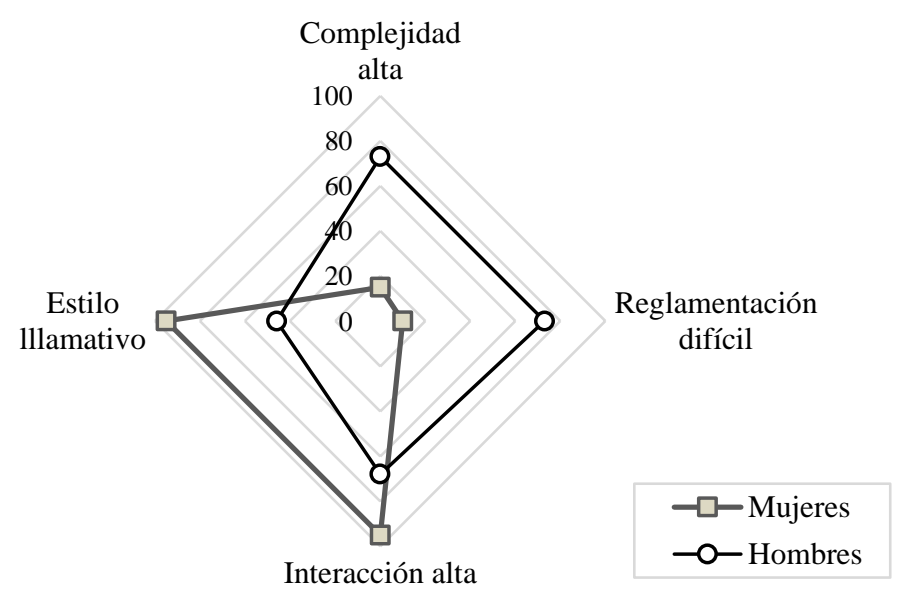

Figura 2. Valoración de las mecánicas de juego según sexo (\%). Elaboración propia (2021)

\section{Preferencias estudiantiles por juegos de mesa}

De acuerdo con los resultados del análisis conjunto, los atributos más valorados en orden decreciente fueron: nivel de interacción $(33,1 \%)$, nivel de complejidad $(24,4 \%)$, formato de juego $(23,2 \%)$ y tiempo de dedicación (19,3\%). Específicamente el estudiantado se inclina por juegos de mesa con alta interacción entre participantes $(0,47)$, de tipo colaborativo $(0,3)$, con un tiempo de duración de 90 minutos o menos $(0,5)$, y con un nivel de complejidad medio $(0,39)$. Ver Figura 3.

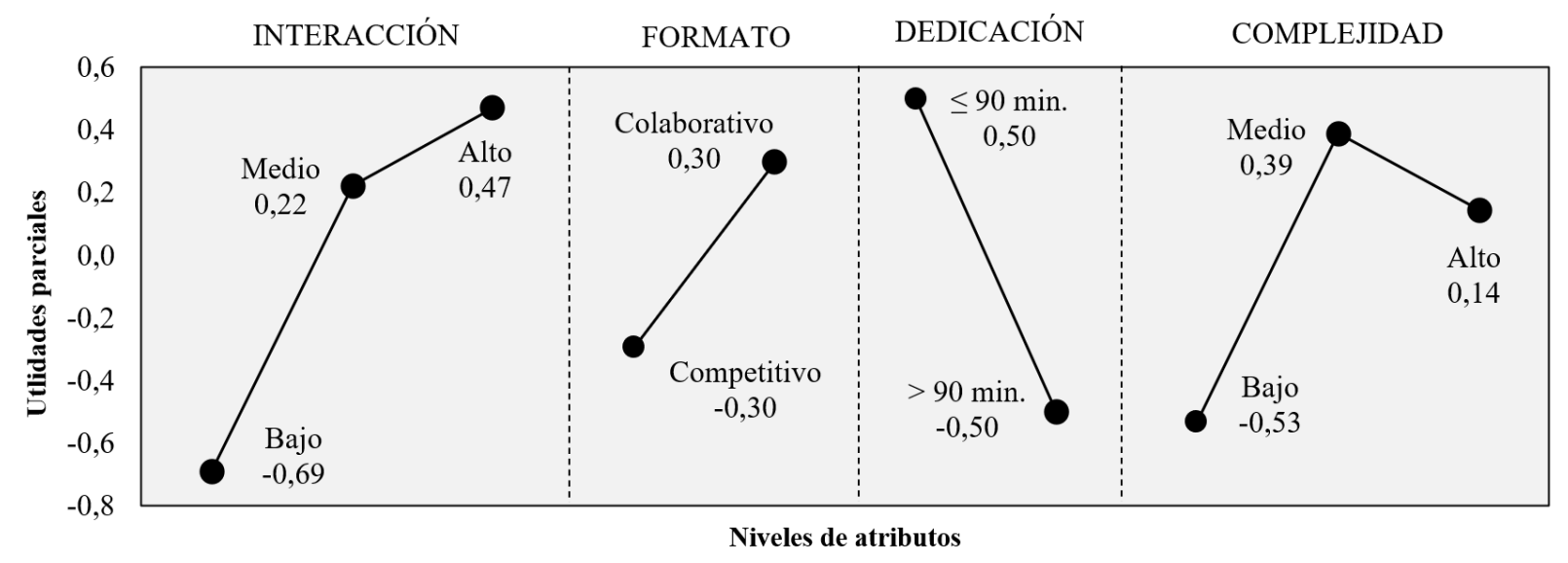

Figura 3. Utilidades parciales por atributo de juego. Elaboración propia (2021) 
El contraste de las preferencias del alumnado por juegos de mesa, según sexo, permitió determinar que no existen diferencias relevantes en cuanto a la importancia relativa del nivel de interacción (33\% para hombres y mujeres). Sin embargo, se halló que para las mujeres es más importante el formato de juego que para los hombres (27\% versus 20\%). Mientras que para los hombres es más relevante la complejidad (26\% versus $23 \%$ ) y el tiempo de dedicación (22\% versus 17\%). Véase la Figura 4.

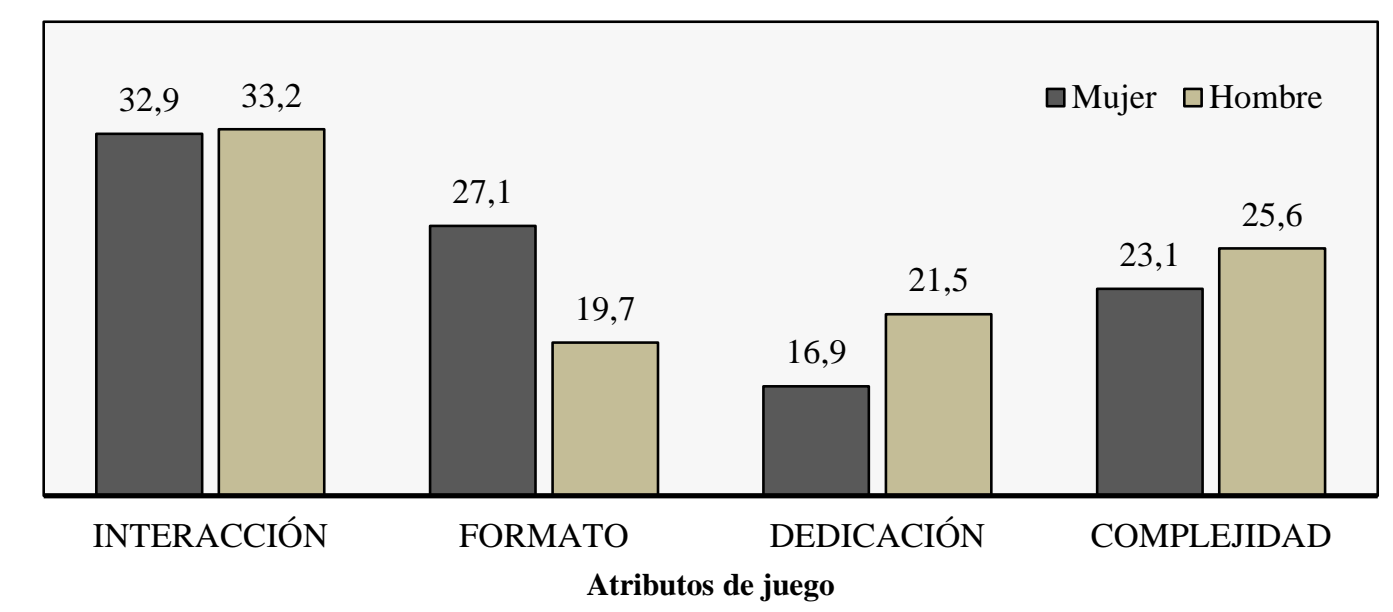

Figura 4. Atributos de juegos de mesa según sexo. Elaboración propia (2021)

Por lo demás, la revisión de las utilidades parciales develó diferencias entre los niveles preferidos entre estudiantes (véase la Tabla 3). Los hombres prefieren juegos de gran nivel de interacción $(0,59)$, competitivos $(0,07)$ y de alta complejidad $(0,35)$. Mientras que las mujeres prefieren juegos de interacción media-alta $(0,35-0,33)$, de tipo colaborativo $(0,7)$ y de complejidad media $(0,63)$. 
Tabla 3

Utilidades parciales por atributo de juego y medidas de ajuste según sexo

\begin{tabular}{llcr}
\hline Atributo & Nivel & Mujer & Hombre \\
\hline Nivel de interacción & Bajo & $-0,683$ & $-0,697$ \\
& Medio & 0,350 & 0,106 \\
Formato de juego & Alto & 0,333 & 0,591 \\
& Competitivo & $-0,700$ & 0,068 \\
Tiempo de dedicación & Colaborativo & 0,700 & $-0,068$ \\
& Una clase & 0,588 & 0,420 \\
& Más de una & $-0,588$ & $-0,420$ \\
Nivel de complejidad & clase & & \\
& Bajo & $-0,550$ & $-0,515$ \\
& Medio & 0,633 & 0,167 \\
(Constante) & Alto & $-0,083$ & 0,348 \\
R de Pearson & & 5,038 & 4,837 \\
Tau de Kendall & & 0,994 & 0,934 \\
\hline
\end{tabular}

Fuente: Elaboración propia (2021)

\section{Discusión y conclusiones}

El objetivo de este trabajo fue analizar las preferencias y actitud del alumnado por los juegos de mesa para la enseñanza de la asignatura gestión en recursos humanos. Los resultados revelan que existe un alto interés de los estudiantes por participar de actividades lúdicas y metodologías activas de enseñanza y aprendizaje. En particular, en línea con lo expuesto por otros autores, se percibe que los juegos de mesa potencian habilidades para la resolución de problemas, las habilidades analíticas y las habilidades para la toma de decisiones (Chacón, 2008; Gómez, 2020; Hernández-Horta et al., 2018; Taspinar et al., 2016). 
Además, se determinó que los atributos de juego que explican sus preferencias son, en orden decreciente, el nivel de interacción entre jugadores, la complejidad del juego, el formato de juego y el tiempo de dedicación. Concretamente, en concordancia con la hipótesis de investigación, los estudiantes prefieren los juegos de mesa con alta interacción entre participantes, de tipo colaborativo, con un tiempo de dedicación de una clase (90 minutos o menos) y con un nivel de complejidad medio.

También, cabe destacar la disposición favorable, de parte del alumnado, por utilizar los juegos de mesa como herramienta educativa para la enseñanza de la asignatura. Hallazgo que coincide con lo concluido por la reciente revisión sistemática de la literatura de Pegalajar (2021) en la cual confirma el interés estudiantil hacia el desarrollo de experiencias didácticas innovadoras basadas en la gamificación. Esto resulta, al mismo tiempo, esencial si se tiene en cuenta que la actitud del estudiantado es un componente clave para el aprendizaje (predisposición por aprender). De este modo, la utilización de los juegos de mesa, como elemento didáctico, motivaría al alumnado y acrecentaría una actitud positiva, impactando también favorablemente el aprendizaje (Sánchez, 2015). Como indican Goudas et al. (2009) las actitudes de los educandos y educandas hacia los juegos son, también, un determinante importante del rendimiento y los logros en las actividades de aprendizaje basadas en juegos.

En relación con los atributos de juego de mesa preferidos cabe denotar que el alumnado valora el nivel de interacción entre participantes más que cualquier otra característica de juego, por lo que debe prestarse especial atención en seleccionar aquellas alternativas que fomenten una alta participación entre jugadores, tal como ha sido destacado por otros autores (Swartout y van Lent, 2003). En este sentido, cabe indicar que la interacción no necesariamente debe ser antagónica, sino que puede ser cooperativa o colaborativa, enfocada en la búsqueda de un beneficio común (Sung y Hwang, 2013). Poy-Castro et al. (2015) en sintonía con lo anterior, destacan las preferencias del alumnado por involucrarse en actividades de equipo que requieran de colaboración y comunicación entre los jugadores. Además, se supone que en función de la naturaleza de la asignatura, gestión de recursos humanos, resultaría idóneo el uso de este tipo de juegos, que requieren de una comunicación más precisa con otros y resoluciones de conflicto más constructivas (Johnson y Johnson, 2009).

No obstante, es relevante, advertir que el aprendizaje colaborativo exitoso depende de la calidad de la interacción social entre los participantes (Järvelä et al., 2008). Ello involucra explorar los sentimientos del estudiantado hacia el trabajo grupal, ya que no solo representa un indicativo 
clave de la calidad de las interacciones sociales grupales, sino que también contribuye al diseño y la aplicación misma de las actividades de aprendizaje colaborativo (Merrell, 2001). También, los hallazgos develan que la elección apropiada del nivel de complejidad y del tiempo afectan positivamente las preferencias por un juego. Esto debido, por un lado, a que la facilidad de uso posibilita que el estudiantado gaste menos tiempo en entender la mecánica y las reglas del juego, para enfocarse en el análisis y toma de decisiones relevantes, y, por otro, una duración más acotada evita que se pierda el interés al practicarlo (Contreras y Eguia, 2016; Tao et al., 2009; Urquidi y Calabor, 2014; Zeng et al., 2017).

Complementariamente, cabe notar que no pueden obviarse las características propias del grupo de trabajo y su contexto de origen, pues rasgos como el género, edad, lugar de procedencia, ingresos, entre otras, influirían en las dinámicas de trabajo (Curlango et al., 2014; Greenberg et al., 2010; Lukosch et al., 2017). Por ejemplo, existe evidencia que sostiene que los grupos con equilibrio de género, muestran un logro significativamente mayor con los juegos colaborativos (Baek y Touati, 2020). Kimura (2004) incluso detectó diferencias biológicas (destrezas) entre hombres y mujeres, que tienen implicancias importantes para el desarrollo de los juegos.

En esta línea, este estudio halló divergencias relevantes entre las preferencias por los juegos de mesa según el sexo del alumnado. Las mujeres, en contraposición a los hombres, se inclinan por juegos de menor complejidad, menos competitivos, con reglamentos sencillos y componentes de juego de buen diseño. Preferencias que han sido evidenciadas también por otros estudios (Hartmann y Klimmt, 2006; Hou, 2012; Lucas y Sherry, 2004; Nakamura y Wirman, 2010). Teniendo en cuenta tales discrepancias, parece importante que el profesorado que enseña la asignatura gestión en recursos humanos y que desee incorporar los juegos como estrategia educativa, incluyan actividades que promuevan la interacción entre estudiantes, pero atendiendo a las diferencias existentes en sus predilecciones. Por tanto, la dificultad (menor complejidad) y diseño de los juegos de mesa (estilo atractivo) serían variables determinantes a considerar, sobre todo en aquellos cursos que posean una participación considerable de mujeres. Como ya lo expusieron Jaramillo y Castellón (2012) es fundamental que exista una relación controlada entre los retos que se exponen al alumnado y la capacidad de estos para sobrellevarlos, pues impactan significativamente la motivación por el aprendizaje. Si un reto es demasiado fácil, provocará aburrimiento, mientras que un reto inalcanzable supondrá la frustración del estudiantado.

En este contexto, es posible advertir alternativas de juegos de mesa que reúnen los atributos destacados por el alumnado y que podrían utilizarse en la asignatura de gestión de recursos 
humanos. Por ejemplo, para el desarrollo de habilidades analíticas, sociales y de resolución de problemas resaltan juegos como la isla prohibida, pandemia y el desierto prohibido. En general, estos juegos de tipo cooperativo plantean una situación crítica que debe ser resuelta en base al trabajo mancomunado de sus participantes, potenciando de este modo competencias como el liderazgo, el trabajo en equipo, la creatividad y la comunicación efectiva.

A modo de reflexión, cabe destacar que para que la enseñanza basada en la aplicación de los juegos de mesa resulte efectiva, debe ser diseñada en función de los propósitos y resultados esperados del curso o materia (Corchuelo-Rodriguez, 2018). No debe ser un evento aislado, cuyo valor descanse en su parte lúdica (divertimento y deleite), lo que pudiese afectar negativamente el nivel académico o la percepción discente sobre la exigencia de la asignatura (Ripoll, 2016).

En tal sentido, para garantizar un aprendizaje efectivo, el profesorado debe ser capaz de vincular apropiadamente la teoría con la práctica del juego (Ahmad et al., 2018; Londoño, 2018) y mantener el interés del estudiantado durante todo el proceso de enseñanza y aprendizaje (Yunyongying, 2014). Por tanto, la planificación de la actividad debiera acompañarse de otras estrategias metodológicas activas y criterios de evaluación integrales, que sirvan a objetivos múltiples de desarrollo del alumnado (Welsh et al., 2016). Ello implica, la consideración y valoración de los procesos, mediante una mirada crítica y reflexiva, que vaya más allá de los resultados propios del juego.

Finalmente, para futuras investigaciones se sugiere: (1) evaluar la importancia relativa de otros atributos básicos para la generación e implementación de un juego, como el espacio físico en que se realiza, el tipo particular de juego aplicado (juegos de dados, de cartas, tablero), el foco del juego (estratégico, de rol, de acción, etc.) y la plataforma de desarrollo del juego, como por ejemplo los juegos virtuales; (2) medir el efecto de ciertas variables de control como la edad, el nivel socioeconómico y las características familiares (influencia) que podrían afectar la predilección y actitud hacia los juegos; (3) replicar este estudio en otras asignaturas o carreras universitarias, de modo de contrastar los resultados y establecer aquellos atributos, de carácter más permanente, en la determinación de las preferencias por juegos de mesa en el estudiantado; y (4) profundizar, bajo una perspectiva cualitativa, respecto de las diferencias detectadas entre los atributos de juego predilectos, según sexo. 


\section{Referencias}

Ahmad, N., Halim, H., Ramayah, T., Popa, S., \& Papa, A. (2018). The ecosystem of entrepreneurial university: the case of higher education in a developing country. International Journal of Technology Management, 78(1/2), 52. https://doi.org/10.1504/ijtm.2018.093935

Araya-Pizarro, S. (2019). Competencias genéricas de los estudiantes de Auditoría requeridas por las Big Four en Chile. Cuadernos de Contabilidad, 20(49), 1-16. https://doi.org/10.11144/javeriana.cc20-49.cgea

Baek, Y., \& Touati, A. (2020). Comparing Collaborative and Cooperative Gameplay for Academic and Gaming Achievements. Journal of Educational Computing Research, 57(8), 2110-2140. https://doi.org/10.1177/0735633118825385

Beneitone, P., Cesar, E., Gonzalez, J., Maleta, M., Siufi, R., \& Wagenaar, G. (2007). Reflexiones y perspectivas de la educación superior en América Latina. Tuning Lationamérica. http://tuningacademy.org/wpcontent/uploads/2014/02/TuningLAIII_Final-Report_SP.pdf

Burke, B. (2016). Gamify: How Gamification Motivates People to Do Extraordinary Things. Routledge.

Cerda, G., Romera, E. M., Casas, J. A., Pérez, C., \& Ortega-Ruiz, R. (2017). Influencia de variables cognitivas y motivacionales en el rendimiento académico en matemáticas en estudiantes chilenos. Educacion XX1, 20(2), 365-385. https://doi.org/10.5944/educXX1.12183

Chacón, P. (2008). El Juego Didáctico como estrategia de enseñanza y aprendizaje ¿Cómo crearlo en el aula? Nueva Aula Abierta, 5(16), 1-8. https://www.academia.edu/download/37885767/juego.pdf

Contreras, R., \& Eguia, J. L. (2016). Gamificación en aulas universitarias. Institut de la Comunicació, Universitat Autònoma de Barcelona. https://core.ac.uk/download/pdf/78545392.pdf

Corchuelo-Rodriguez, C. A. (2018). Gamificación en educación superior: experiencia innovadora para motivar estudiantes y dinamizar contenidos en el aula. Edutec. Revista Electrónica de Tecnología Educativa, 63, 29. https://doi.org/10.21556/edutec.2018.63.927

Curlango, C. M., Ibarra, J. E., Chávez, G. E., González, M. L., Arredondo, L. E., \& Rodríguez, M. D. (2014). Understanding Game Playing Preferences. CLEI Electronic Journal, 17(3). http://www.scielo.edu.uy/pdf/cleiej/v17n3/v17n3a10.pdf

Daviau, R. (2011). Design Intuitively. En The Kobold Guide to Boardgame design (pp. 42-49). Open Design.

Dichev, C., \& Dicheva, D. (2017). Gamifying education: what is known, what is believed and what remains uncertain: a critical review. International Journal of Educational Technology in Higher Education, 14(9), 1-36. https://doi.org/10.1186/s41239-017-0042-5

Díez, J. C., Bañeres, D., \& Montse, S. (2017). Experiencia de gamificación en Secundaria en el Aprendizaje de Sistemas Digitales. Education in the Knowledge Society (EKS), 18(2), 85. https://doi.org/10.14201/eks201718285105

Esnaola, G., Galli, M., \& Colla, M. de la P. (2017). La formación superior en desarrollo de los videojuegos en la educación argentina. En Actas del V Congreso Internacional de Videojuegos y Educación (CIVE'17). Universidad de La Laguna, España. 
Gómez, J. L. (2020). Gamificación en contextos educativos: analisis de aplicación en un programa de contaduría pública a distancia. Revista Universidad y Empresa, 22(38), 8-39. https://doi.org/10.12804/revistas.urosario.edu.co/empresa/a.6939

González, J. A., Olivares, S. A., García, E., \& Figueroa, I. G. (2017). Propuesta de gamificación en el aula: uso de una plataforma para motivar a los estudiantes del programa académico de informática de la Universidad Autónoma de Nayarit. Educateconciencia, 13(14), 71-79. http://tecnocientifica.com.mx/educateconciencia/index.php/revistaeducate/article/view/306

Goudas, M., Magotsiou, E., \& Hatzigeorgiadis, A. (2009). Psychometric Properties of the Greek Version of the Feelings Toward Group Work Questionnaire. European Journal of Psychological Assessment, 25, 204-210. https://doi.org/10.1027/1015-5759.25.3.204

Greenberg, B. S., Sherry, J., Lachlan, K., Lucas, K., \& Holmstrom, A. (2010). Orientations to video games among gender and age groups. Simulation and Gaming, 41(2), 238-259. https://doi.org/10.1177/1046878108319930

Hair, J., Black, W., Babin, B., \& Anderson, R. (2014). Multivariate Data Analysis. Pearson Education Limited. https://doi.org/10.1007/978-3-319-01517-0_3

Hamari, J., Koivisto, J., \& Sarsa, H. (2014). Does gamification work? - A literature review of empirical studies on gamification. Proceedings of the Annual Hawaii International Conference on System Sciences, 3025-3034. https://doi.org/10.1109/HICSS.2014.377

Hartmann, T., \& Klimmt, C. (2006). Gender and computer games: Exploring females' dislikes. Journal of ComputerMediated Communication, 11(4), 910-931. https://doi.org/10.1111/j.1083-6101.2006.00301.x

Hassinger-Das, B., Toub, T. S., Zosh, J. M., Michnick, J., Golinkoff, R., \& Hirsh-Pasek, K. (2017). More than just fun: a place for games in playful learning / Más que diversión: el lugar de los juegos reglados en el aprendizaje lúdico. Infancia y Aprendizaje, 40(2), 191-218. https://doi.org/10.1080/02103702.2017.1292684

Hernández-Horta, I. A., Monroy-Reza, A., \& Jiménez-García, M. (2018). Aprendizaje mediante Juegos basados en Principios de Gamificación en Instituciones de Educación Superior. Formación universitaria, 11(5), 31-40. https://doi.org/10.4067/S0718-50062018000500031

Hou, H. T. (2012). Exploring the behavioral patterns of learners in an educational massively multiple online roleplaying game (MMORPG). Computers and Education, 58(4), 1225-1233. https://doi.org/10.1016/j.compedu.2011.11.015

Jaramillo, O., \& Castellón, L. (2012). Educación y videojuegos. Revista Latinoamericana de Comunicación Chasqui, 117, 11-19. https://doi.org/10.1080/0740817X.2012.757679

Järvelä, S., Järvenoja, H., \& Veermans, M. (2008). Understanding the dynamics of motivation in socially shared learning. International Journal of Educational Research, 47(2), 122-135. https://doi.org/10.1016/j.ijer.2007.11.012

Johnson, D. W., \& Johnson, R. T. (2009). Energizing learning: The instructional power of conflict. Educational Researcher, 38(1), 37-51. https://doi.org/10.3102/0013189X08330540

Kimura, D. (2004). Sexo y capacidades mentales. Ariel.

Lancaster, K. (1966). A New Approach to Consumer Theory. Journal of Political Economy, 74(2), 132-157.

Londoño Cardozo, J. (2018). Educación para emprender ¿práctica o teoría? Revista Argentina de Investigación en Negocios, 4(1), 97-106. http://ppct.caicyt.gov.ar/index.php/rain/article/download/V4n1a8/pdf 
Lucas, K., \& Sherry, J. L. (2004). Sex differences in video game play: A communication-based explanation. Communication Research, 31(5), 499-523. https://doi.org/10.1177/0093650204267930

Lukosch, H., Kurapati, S., Groen, D., \& Verbraeck, A. (2017). Gender and cultural differences in game-based learning experiences. Electronic Journal of e-Learning, 15(4), 310-319.

Melo, M. P., \& Hernández, R. (2014). El juego y sus posibilidades en la enseñanza de las ciencias naturales. Innovación educativa, 14(66), 41-64. http://www.scielo.org.mx/pdf/ie/v14n66/v14n66a4.pdf

Merrell, K. W. (2001). Assessment of Children's Social Skills: Recent Developments, Best Practices, and New Directions. Exceptionality: A Special Education Journal, 9(1-2), 3-18. https://doi.org/10.1080/09362835.2001.9666988

Nakamura, R., \& Wirman, H. (2010). Girlish Counter-Playing Tactics. Game Studies, 5(1). http://www.gamestudies.org/0501/nakamura_wirman/

Ortiz-Colón, A.-M., Jordán, J., \& Agredal, M. (2018). Gamificación en educación: una panorámica sobre el estado de la cuestión. Educacao e Pesquisa, 44, 1-17. https://doi.org/10.1590/S1678-4634201844173773

Pegalajar, M. del C. (2021). Implicaciones de la gamificación en Educación Superior: una revisión sistemática sobre la percepción del estudiante. Revista de Investigación Educativa, 39(1), 169-188. https://doi.org/10.6018/rie.419481

Pérez, Q. (2016). Aplicación de herramientas de gamificación en física y química de secundaria. Opción.

Poy-Castro, R., Mendaña-Cuervo, C., \& González, B. (2015). Diseño y evaluación de un juego serio para la formación de estudiantes universitarios en habilidades de trabajo en equipo. RISTI - Revista Iberica de Sistemas e Tecnologias de Informacao, spe3, 71-83. https://doi.org/10.17013/risti.e3.71-83

Ripoll, O. (2016). Taller de Creación de JOCS", una asignatura gamificada. En R. Contreras \& J. L. Eguia (Eds.), Gamificación en aulas universitarias (pp. 25-37). Institut de la Comunicació, Universitat Autònoma de Barcelona. https://core.ac.uk/download/pdf/78545392.pdf

Rogerson, M. J., Gibbs, M. R., \& Smith, W. (2018). Cooperating to Compete: the Mutuality of Cooperation and Competition in Boardgame Play. Proceedings of the 2018 CHI Conference on Human Factors in Computing Systems, 1-13. https://doi.org/10.1145/3173574.3173767

Sánchez, F. J. (2015). Gamificación. Education in the Knowledge Society (EKS), 16(2), 13. https://doi.org/10.14201/eks20151621315

Sánchez, G. (2010). Las estrategias de aprendizaje a través del componente lúdico. marcoELE. Revista de Didáctica Español Lengua Extranjera, 11, 1-68. http://www.redalyc.org/articulo.oa?id=92152537016

Silva, J., \& Maturana, D. (2017). Una propuesta de modelo para introducir metodologías activas en educación superior. Innovación Educativa, 17(73), 117-131. http://www.scielo.org.mx/pdf/ie/v17n73/1665-2673-ie17-73-00117.pdf

Sung, H. Y., \& Hwang, G. J. (2013). A collaborative game-based learning approach to improving students' learning performance in science courses. Computers and Education, 63, 43-51. https://doi.org/10.1016/j.compedu.2012.11.019

Swartout, W., \& van Lent, M. (2003). Making a game of system design. Communications of the ACM, 46(7), 32-39. https://doi.org/10.1145/792704.792727 
Tao, Y. H., Cheng, C. J., \& Sun, S. Y. (2009). What influences college students to continue using business simulation games? The Taiwan experience. Computers and Education, 53(3), 929-939. https://doi.org/10.1016/j.compedu.2009.05.009

Taspinar, B., Schmidt, W., \& Schuhbauer, H. (2016). Gamification in education: A board game approach to knowledge acquisition. Procedia Computer Science, 99, 101-116. https://doi.org/10.1016/j.procs.2016.09.104

Urquidi, A., \& Calabor, M. (2014). Aprendizaje a través de juegos de simulación: un estudio de los factores que determinan su eficacia pedagógica. Edutec: Revista electrónica de tecnología educativa, 0(47), 1-15. https://doi.org/10.21556/edutec.2014.47.75

Victoria-Uribe, R., Utrilla-Cobos, S., \& Santamaría-Ortega, A. (2017). Diseño de juegos de mesa. Una introducción al tema con enfoque para diseñadores industriales. Revista Legado de Arquitectura y Diseño, 21, 98-107. https://legadodearquitecturaydiseno.uaemex.mx/article/view/9376/7863

Welsh, D. H. B., Tullar, W. L., \& Nemati, H. (2016). Entrepreneurship education: Process, method, or both? Journal of Innovation \& Knowledge, 1(3), 125-132. https://doi.org/10.1016/j.jik.2016.01.005

Yunyongying, P. (2014). Gamification: Implications for Curricular Design. Journal of Graduate Medical Education, 6(3), 410-412. https://doi.org/10.4300/jgme-d-13-00406.1

Zagal, J. P., Rick, J., \& Hsi, I. (2006). Collaborative games: Lessons learned from board games. Simulation and Gaming, 37(1), 24-40. https://doi.org/10.1177/1046878105282279

Zeng, Z., Tang, J., \& Wang, T. (2017). Motivation mechanism of gamification in crowdsourcing projects. International Journal of Crowd Science, 1(1), 71-82. https://doi.org/10.1108/ijcs-12-2016-0001

Zepeda-Hernández, S., Abascal-Mena, R., \& López-Ornelas, E. (2016). Intregación de gamificación y aprendizaje activo en el aula. Ra Ximhai, 12(6), 315-325. http://www.redalyc.org/pdf/461/46148194022.pdf

\section{Contribución autoral}

a) Concepción y diseño del trabajo; b) Adquisición de datos; c) Análisis e interpretación de datos; d) Redacción del manuscrito; e) revisión crítica del manuscrito.

S. C. A-P. ha contribuido en a, b, c, d, e.

\section{Editora científica responsable}

Mag. Florencia de León 